\title{
Exceptional points of an endomorphism of the projective plane
}

\author{
E. Amerik and F. Campana
}

October 15, 2003

\section{Introduction}

Let $f: \mathbb{P}^{k} \rightarrow \mathbb{P}^{k}$ be an endomorphism of the complex projective space, of degree bigger than one. An algebraic subset $V \in \mathbb{P}^{k}$ is called completely invariant for $f$, if $f^{-1}(V)=V$. The exceptional set of $f$ is the largest completely invariant proper algebraic subset of $\mathbb{P}^{k}$ (it is observed for example in $[\mathrm{BD}$ that this definition does make sense).

It seems to be interesting from the point of view of dynamics to understand which algebraic subsets of $\mathbb{P}^{k}$ can actually be exceptional or completely invariant. Recently, this question was studied by several authors ([FS], CLN], BCS] $)$. For example, in [BCS] it is shown that an exceptional set is always a union of linear subspaces. It is easy to see (and observed in [FS]) that there are at most $k+1$ hyperplanes in an exceptional set. This bound is of course sharp: take $f$ raising the homogeneous coordinates to $m$ th power. However, no such bound was found, up to now, for the number of higher-codimensional components of an exceptional set. In this article, we first treat the simplest case of this problem: that of points in $\mathbb{P}^{2}$, and then show how to obtain a bound for the number of completely invariant linear subspaces of codimension two.

More precisely, let $f: \mathbb{P}^{2} \rightarrow \mathbb{P}^{2}$ be an endomorphism of degree bigger than one. Let us say that $f$ is completely ramified at a point $P \in \mathbb{P}^{2}$, if $f^{-1}(P)$ consists of one point. In Section 3, we prove the following

Theorem 1: $f$ can be completely ramified at nine points at most. No three such points are on a line and no six on a conic. Moreover, if $f$ is completely ramified at nine points, they lie on a unique cubic, which has a singularity at one of them.

Our result does not seem to be the best possible. Indeed, we do not know any example of an $f$ which is completely ramified at more than three points (an obvious $f$ completely ramified at three points is given by the formula

$$
f(x: y: z)=\left(x^{m}: y^{m}: z^{m}\right)
$$


one can also take maps of the type

$$
f(x: y: z)=\left(x^{m}: y^{m}: z^{m}+x y g(x, y, z)\right)
$$

and compositions of such).

Let us now explain the idea of the proof of this theorem. Let $m$ be such that $f^{*}\left(\mathcal{O}_{\mathbb{P}^{2}}(1)\right)=\mathcal{O}_{\mathbb{P}^{2}}(m)$, so that $\operatorname{deg}(f)=m^{2}$. Suppose that $f$ is completely ramified at $3 d$ points $P_{1}, \ldots, P_{3 d}$ lying on a smooth curve $D$ of degree $d$, and consider the (rarely occuring of course) case when the inverse image $C$ of $D$ is smooth and reduced. By adjunction, we have $\operatorname{deg}\left(K_{C}\right)=m d(m d-3)$. On the other hand, at each $P_{i}$, the ramification index of $\left.f\right|_{C}: C \rightarrow D$ is $m^{2}-1$, and by Hurwitz' formula,

$$
\operatorname{deg}\left(K_{C}\right) \geq \operatorname{deg}\left(\left(\left.f\right|_{C}\right)^{*} K_{D}\right)+3 d\left(m^{2}-1\right)
$$

so that

$$
m d(m d-3) \geq m^{2} d(d-3)+3 d\left(m^{2}-1\right),
$$

and this is impossible if $m>1$.

Now through any nine points in the plane there is a cubic. This cubic, and a fortiori its inverse image, does not have to be smooth. But it happens that one can, with some modifications, make the above argument work for singular $D$ and $C$, replacing the canonical class by the dualizing sheaf. One obtains then that there are at most $3 d-1$ points of complete ramification in the smooth locus of any curve of degree $d$ in $\mathbb{P}^{2}$, and also at most $3 d-1$ points of complete ramification on any curve which does not contain a component of the branch locus. This implies almost immediately that $f$ can be completely ramified at 11 points at most, and some further analysis implies our theorem. An important point of this analysis is that a point of complete ramification must be of very high multiplicity on the direct image of the ramification divisor (see Proposition 1 below).

In the rest of the paper, we generalize this to higher dimension. We obtain

Theorem 2: Let $f: \mathbb{P}^{N-1} \rightarrow \mathbb{P}^{N-1}$ be a morphism of degree bigger than one. The number of codimension-two completely invariant subspaces for $f$ is less than $4 N^{2}$.

The proof of this theorem goes essentially along the same lines. One considers a general $\mathbb{P}^{2}$ in $\mathbb{P}^{N-1}$, the intersection points $P_{i}$ of our completely invariant subspaces with this $\mathbb{P}^{2}$ and the restriction of $f$ to the inverse image of this $\mathbb{P}^{2}$. One obtains this time (Proposition 3) that a curve of degree $d$ contains at most $N d-1$ of the points $P_{i}$ in its smooth locus, and a "flexible" curve of degree $d$ contains at most $N d-1$ of the points $P_{i}$. Again, this implies almost immediately a somewhat weaker bound $6 N^{2}$. We can improve this to $4 N^{2}$, either by recalling a Cayley-Bacharach type statement due to Ellia and Peskine (a remark in the end of the section 4), or by proving a general combinatorial result on points of high multiplicity on an effective divisor in the plane (Section 5), which in particular implies our Theorem 
2. Again, our bound is certainly not the best possible; it is however clear that $N^{2}$ must appear, as the map raising the coordinates to the $m$ th power has $\frac{1}{2} N(N-1)$ completely invariant subspaces of codimension 2 .

We are grateful to N. Sibony, S. Cantat, T.-C. Dinh and C. Favre for asking the question, and to A. Otwinowska and D. Perrin for providing us with some useful references on the Hilbert function of a set of points in the plane.

\section{Some remarks on differential forms}

Let us first recall a few basic facts on the dualizing sheaf of a curve on a smooth surface (see for example [BPV], chapter II), and make some observations on the behaviour of this sheaf under morphisms.

Let $D \subset S$ be an effective divisor on a smooth surface $S$. The dualizing sheaf $\omega_{D}$ is then the line bundle $\left.\mathcal{O}\left(K_{S}+D\right)\right|_{D}$. If $D$ is reduced, then $\omega_{D}$ identifies with a sheaf of meromorphic differentials on the normalization $\tilde{D}$, called Rosenlicht differentials. Concretely, these are the differentials $\phi$ such that for any $x \in D$ and $g \in \mathcal{O}_{D, x}$, one has

$$
\sum_{y \in n^{-1}(x)} \operatorname{res}(y, g \phi)=0,
$$

where $n$ is the normalization map. Moreover one can describe $\omega_{D}$ in local coordinates in the following way: if a local equation of $D$ in $S$ is $F(x, y)=0$, and $x$ is nonconstant on any local branch of $D$, then $\omega_{D}$ is locally generated by $\frac{d x}{F_{y}^{\prime}}$ (or, more precisely, by $n^{*}\left(\frac{d x}{F_{y}^{\prime}}\right)$ ), which one views as the "residue" of $\frac{d x \wedge d y}{F}$.

Lemma 1: Let $f: X \rightarrow Y$ be a finite morphism of smooth surfaces, $D \subset Y$ a (reduced) curve and $C=f^{*} D$ the (scheme-theoretic) inverse image of $D$. Then $f$ induces the injection of sheaves $\left(\left.f\right|_{C_{\text {red }}}\right)^{*} \omega_{D} \rightarrow \omega_{C_{\text {red }}}$.

Proof: Let $C=\sum_{i=1}^{l} n_{i} C_{i}$ be the decomposition of the divisor $C$ into the sum of its reduced irreducible components, so that the ramification divisor of $f$ is $R=$ $\sum_{i=1}^{l}\left(n_{i}-1\right) C_{i}+R^{\prime}$, where $R^{\prime}$ contains no component of $C$. The map $f$ induces the injection $f^{*} K_{Y} \rightarrow K_{X}$, locally given by the multiplication by the Jacobian. The image of $f^{*} K_{Y}$ is of course contained in the subsheaf of forms vanishing on $C_{i}$ with multiplicity $n_{i}-1$, so we get an injection $f^{*} K_{Y} \rightarrow K_{X}-\sum_{i=1}^{l}\left(n_{i}-1\right) C_{i}$. Adding $C$ and restricting to $C_{r e d}$, we get a morphism $\left(\left.f\right|_{C_{r e d}}\right)^{*} \omega_{D} \rightarrow \omega_{C_{r e d}}$, which is still an injection as $C$ and $R^{\prime}$ do not have common components.

Remarks: 1) It is clear that in general, a morphism $h: C \rightarrow D$, even a surjective one, does not induce a morphism of the dualizing sheaves when $D$ is not smooth (take for example the normalization map of a cuspidal curve).

2) The cokernel of our injection is the structure sheaf of the intersection of $R^{\prime}$ and $C$, so that the contribution of each "ramification point" to the difference of 
$\operatorname{deg}\left(\omega_{C_{r e d}}\right)$ and $\operatorname{deg}\left(\left(\left.f\right|_{C_{r e d}}\right)^{*} \omega_{D}\right)$ is the intersection index of $R^{\prime}$ and $C$ at that point.

3) One could also write all this in local coordinates using the above explicit description of the dualizing sheaf of a reduced curve.

4) Obviously we also have a morphism from $\left(\left.f\right|_{C}\right)^{*} \omega_{D}$ to $\omega_{C}$, and it is not injective when $C$ is non-reduced (neither is its cokernel concentrated at points).

We shall need a local computation in order to estimate the "ramification multiplicities" at the points of complete ramification. Let now $(D, 0)$ be a germ of a smooth curve, $z$ a coordinate on $D$. Let $(C,(0,0))$ be a germ of a reduced curve on a smooth surface, given by the equation $F(x, y)=0$. Let further $n: \tilde{C} \rightarrow C$ be the normalization of $C$, and $Q_{1}, \ldots, Q_{r}$ the points of $\tilde{C}$ with $n\left(Q_{i}\right)=(0,0)$.

Lemma 2: Let $g:(C,(0,0)) \rightarrow(D, 0)$ be a germ of a finite map of local degree $\delta$ at $(0,0)$. Denote by $\mu_{i}$ the vanishing order at $Q_{i}$ of $n^{*} g^{*} d z$ as a section of $n^{*} \omega_{C}$. Then $\sum_{i=1}^{r} \mu_{i} \geq \delta-1$.

Proof: Let $e$ be the degree of the restriction to $C$ of the first projection $p_{1}$ : $(x, y) \rightarrow x$. We may suppose that the function $F(x, y)$ defining $C$ is in the Weierstrass form:

$$
F(x, y)=y^{e}+a_{e-1}(x) y^{e-1}+\ldots+a_{1}(x) y+a_{0}(x),
$$

with $a_{i}(0)=0$ for any $i$.

Suppose first that $C$ is an irreducible germ. Choose a coordinate $t$ on $\tilde{C}$ so that the map $p_{1} \cdot n$ is given by $x=t^{e}$ and the map $g \cdot n$ is given by $z=h(t) t^{\delta}$, where $h$ is holomorphic with $h(0) \neq 0$. We have then $n^{*} d x=e t^{e-1} d t$ and $n^{*} g^{*} d z=h_{1}(t) t^{\delta-1} d t$ with $h_{1}$ holomorphic.

As $n^{*} \omega_{C}$ is generated by $n^{*}\left(\frac{d x}{F_{y}^{\prime}}\right)$, all that we have to show is that $n^{*} g^{*} d z=$ $h_{2}(t) t^{\delta-1} n^{*}\left(\frac{d x}{F_{y}^{\prime}}\right)$ for some holomorphic $h_{2}$, i.e. that $n^{*} F_{y}^{\prime}$ has a zero of order at least $e-1$ at 0 . But this is obvious, as $n^{*} y$ has a zero at 0 , and $n^{*} x$ has a zero of order $e$.

Now if $C$ is reducible, let $C_{j}, j=1, \ldots, l$ be the irreducible components and $F(x, y)=\prod_{j=1}^{l} F_{j}(x, y)$, each $F_{j}$ defining $C_{j}$. Denote by $n_{j}: \tilde{C}_{j} \rightarrow C_{j}$ the normalization and by $\delta_{j}$ the degree of $\left.g\right|_{C_{j}}$. As $\delta$ is the sum of $\delta_{j}$, it suffices to show that $g^{*} n_{j}^{*} d z$ has a zero of order at least $\delta_{j}$ at $n_{j}^{-1}(0,0)$, again as a section of $n^{*} \omega_{C}$, of course.

This is the same kind of computation as before: choose appropriate coordinates, write

$$
F_{j}(x, y)=y^{e_{j}}+a_{j, e_{j}-1}(x) y^{e_{j}-1}+\ldots+a_{j, 1}(x) y+a_{j, 0}(x),
$$

$e_{j}$ being the degree of $p_{1} \cdot n_{j}$. Repeating the previous considerations, we see that this time we have to show that $n_{j}^{*} F_{y}^{\prime}$ has a zero of order at least $e_{j}$ at 0 . But $F=F_{j} G$ with $G$ holomorphic and vanishing at $(0,0)$ and $F_{y}^{\prime}=\left(F_{j}\right)_{y}^{\prime} G+F_{j} G_{y}^{\prime}$. We have that $n_{j}^{*}\left(F_{j}\right)_{y}^{\prime}$ has a zero of order at least $e_{j}-1, n_{j}^{*} F_{j}$ is identically zero, and $n_{j}^{*} G$ has a zero at 0 , which proves our assertion. 
This lemma, together with the projection formula, implies the following fact which will be quite useful:

Proposition 1: Let $f: X \rightarrow Y$ be a finite (proper) morphism between smooth surfaces (or their germs). Let $R$ denote its ramification divisor. For any $y \in Y$, the following inequality holds:

$$
\operatorname{mult}_{y}\left(f_{*} R\right) \geq \sum_{x \in f^{-1}(y)}\left(\delta_{x}(f)-1\right),
$$

where mult $_{y}$ stands for the multiplicity at $y$ and $\delta_{x}(f)$ denotes the local degree of $f$ at $x$.

Proof: Choose a sufficiently general smooth curve $D$ through $y$, so that mult $_{y}\left(f_{*} R\right)$ equals to the local intersection index $\left(f_{*} R \cdot D\right)_{y}$. By the projection formula, this intersection index is equal to $\sum_{x \in f^{-1}(y)}\left(R \cdot f^{*} D\right)_{x}$. It remains to apply Lemma 2 together with the second remark after Lemma 1.

Finally, we prove one more lemma about differentials on singular curves, though it is not absolutely necessary for the sequel:

Lemma 3: Let $g:\left(\mathbb{C}^{2}, 0\right) \rightarrow\left(\mathbb{C}^{2}, 0\right)$ be a germ of a finite holomorphic map, of local degree $\delta$. Let $(D, 0) \subset\left(\mathbb{C}^{2}, 0\right)$ be a germ of a (reduced) curve, such that $(C, 0)=g^{*}(D, 0)$ is reduced, and let $r$ be the multiplicity of $D$ at 0 . Then the length of the cokernel of the lift to the normalization of $C$ of the injection of the dualizing sheaves $\left(\left.g\right|_{C}\right)^{*} \omega_{D} \rightarrow \omega_{C}$ is at least $r(\delta-1)$.

Proof: Since $C$ is reduced, this length is the intersection multiplicity of $C$ and the ramification divisor $R$, or, by projection formula, the intersection multiplicity of $D$ and $f_{*} R$. This multiplicity is at least equal to the product of the multiplicities at 0 of $D$ and $f_{*} R$, so the lemma follows from Proposition 1.

Remark: The condition that $D$ is smooth is essential for Lemma 2 ( moreover, Lemma 2 does not make sense for a singular $D$, since in this case the inverse image of a Rosenlicht differential on $D$ does not have to be a Rosenlicht differential on $C$ ). Lemma 3 as it is also does not make sense for non-reduced $C$, for example, because in this case the cokernel is not concentrated in points, and also because while a general point of $C$ should not contribute at all to the difference of the degrees of $\left(\left.g\right|_{C}\right)^{*} \omega_{D}$ and $\omega_{C}$, the local degree $\delta$ at such a point is still bigger than one (this however should be possible to repair by considering the local degree of $\left.g\right|_{C_{\text {red }}}$ rather than $\delta$ ). We do not know if there is some analogue of Lemma 3 for non-reduced curves, and it is an interesting question (a suitable analogue could simplify and improve the proof of our theorems). In any case there are many examples showing that if $D$ is singular 
and $C$ is non-reduced, the length of the cokernel of $\left(\left.g\right|_{C_{r e d}}\right)^{*} \omega_{D} \rightarrow \omega_{C_{r e d}}$ need not be bounded from below by a non-trivial function of the local degree of the map from $C_{\text {red }}$ to $D$. We list below several of them, in which this length is zero, because the "residual ramification" $R^{\prime}$ is empty:

\section{Examples:}

1) $g$ given by the formula $u=x^{m}, v=y^{m}$, and $D=\{(u, v): u v=0\}$. The ramification divisor $R$ is given by the equation $x^{m-1} y^{m-1}=0$, and $C_{\text {red }}$ by the equation $x y=0$. Our inclusion $\left(\left.g\right|_{C_{r e d}}\right)^{*} \omega_{D} \rightarrow \omega_{C_{\text {red }}}$ is thus an isomorphism.

2) An example with locally irreducible $D$ : let $m>1$ be an odd integer and let $g$ be given by $u=\left(x^{m}-y^{m}\right) / 2, v=x y$. The equation of the ramification divisor is then $x^{m}+y^{m}=0$. Consider $D=\left\{(u, v): u^{2}+v^{m}=0\right.$. The equation of the inverse image of $D$ is $\left(\left(x^{m}-y^{m}\right) / 2\right)^{2}+(x y)^{m}=\left(x^{m}+y^{m}\right)^{2} / 4=0$, so $g^{*}(D)=2 R$ and $\omega_{C_{r e d}}=\left(\left.g\right|_{C_{r e d}}\right)^{*} \omega_{D}$.

3) A more exotic example with cuspidal $D$ is the following: let $V \subset \mathbb{C}^{3}$ be the canonical hypersurface singularity given by the equation $u^{2}+v^{3}+w^{5}=0$. It is a quotient singularity $h: \mathbb{C}^{2} \rightarrow V=\left(\mathbb{C}^{2} / A_{5}\right)$, for a certain $S l_{2}(\mathbb{C})$-representation of $A_{5}$. The map $h$ is unramified except at the origin. Let $p(u, v, w):=(u, v)$, and $g: \mathbb{C}^{2} \rightarrow \mathbb{C}^{2}$ be the composition $p \circ h$. Let $D$ be given by the equation $u^{2}+v^{3}=0$. Let $C=g^{*}(D)$, and let $R$ be the ramification divisor of $g$. One checks that $R=4 C_{\text {red }}$ and $g^{*}(D)=5 C_{r e d}$, that is, $\omega_{C_{r e d}}=\left(\left.g\right|_{C_{r e d}}\right)^{*} \omega_{D}$. In this example, the degree of $g$ is 300 , and the degree of $g$ restricted to $C_{r e d}$ is 60 . We do not know if there are examples with cuspidal $D$ and arbitrarily high degree of $\left.g\right|_{C_{r e d}}$.

\section{Proof of Theorem 1}

We start with the following

Proposition 2: Let $f$ be an endomorphism of projective plane, of degree bigger than one. Let $D \subset \mathbb{P}^{2}$ be a curve of degree $d$. Then $f$ cannot be completely ramified at $3 d$ (or more) points of the smooth locus of $D$.

Proof: Let $f$ be an endomorphism as above, completely ramified at $3 d$ smooth points of $D$. Let $f^{*} \mathcal{O}(1)=\mathcal{O}(m)$, let $C=f^{*} D$ and let $c$ be the degree of $C_{\text {red }}$. By the projection formula, the degree of the restriction of $f$ to $C_{r e d}$ is $\frac{m c}{d}$. We have, by Lemma 1, the induced injection $\alpha:\left(\left.f\right|_{C_{r e d}}\right)^{*} \omega_{D} \rightarrow \omega_{C_{r e d}}$, and, as we suppose that our $3 d$ points of complete ramification are in the smooth locus of $D$, by Lemma 2 , each of them contributes at least $\frac{m c}{d}-1$ to the length of the cokernel of the lift of this injection to the normalization of $C_{\text {red }}$. So that we have:

$$
\operatorname{deg}\left(\omega_{C_{r e d}}\right) \geq 3 d\left(\frac{m c}{d}-1\right)+\frac{m c}{d} \operatorname{deg}\left(\omega_{D}\right),
$$

that is, 


$$
c(c-3) \geq d m c-3 d,
$$

which of course implies that $c \leq d$, since by the definition of $c$ we have $d m \geq c$, and even that $c<d$, since $m>1$. If moreover $m c-3>0$ we conclude that $c(c-3) \geq c(m c-3)>c(c-3)$, a contradiction. So that we must have $m=2$ and $c=1$. Then $d=2$, since $\frac{m c}{d}$ is an integer (the degree of the restriction of $f$ to $\left.C_{r e d}\right)$. This implies that the restriction of $f$ to $C_{r e d}$ is one-to-one and so $C=4 C_{\text {red }}$. Since the ramification divisor of $f$ is of degree three, it must be $3 C_{\text {red }}$, so that the complement to $C_{\text {red }}$ in $\mathbb{P}^{2}$ is an unramified covering of degree four of the complement to $D$ in $\mathbb{P}^{2}$. This is a contradiction because the fundamental group of the latter is $\mathbb{Z} / 2 \mathbb{Z}$.

Notice that this proposition already implies that an endomorphism $f$ (of degree $>1$ ) of projective plane cannot be completely ramified at more than 11 points, by the following general remark:

Lemma 4: Let $E$ be a subset of the projective plane, such that any curve of degree $1 \leq d \leq 4$ contains at most $3 d-1$ points of $E$ in its smooth locus. Then $E$ cannot contain more than 11 points.

Proof: Suppose that we have 12 points $P_{1}, \ldots, P_{12}$ in $E$. It is immediate from the assumption that no three of those points are on a line, no six on a conic, no ten on a cubic, and through any nine there is a unique cubic which must be singular at one of the $P_{i}$ (but irreducible). Consider the linear system of quartics passing through $P_{1}, \ldots, P_{12}$. It is a linear subspace $L$ of dimension at least two in the projective space $P=\mathbb{P}^{14}$ parametrizing all the plane quartics. Suppose that all these quartics are singular. Then, by Bertini, they are all singular at one of the $P_{i}$, say $P_{1}$. So $L$ is contained in the linear subspace $M \subset P$ parametrizing the quartics singular at $P_{1}$. The space $M$ is of dimension 11. Now consider the subset $V$ of $M$ parametrising reducible quartics which are of the form $Q=H \cup C$ with $H$ a line through $P_{1}$ and $C$ is a (possibly reducible) cubic through $P_{1}$. Clearly $V$ is a subvariety of $M$ of dimension 9 . So $L$ and $V$ must intersect; that is, among our quartics passing through 12 points, there is one which is a union of a line and a cubic. As we have already seen that no three points are on a line and no ten on a cubic, this gives a contradiction. So among our quartics there must be a smooth one, but this again contradicts our assumption.

The proof of Theorem 1 does not depend on Lemma 4:

Proof of Theorem 1: Consider first an endomorphism $f$ with nine points of complete ramification $P_{1}, \ldots, P_{9}$. We see from Proposition 2 that through these points there is a unique irreducible cubic $D$ which has a singularity at one of our points, say, $P_{1}$. Let $R$ denote, as usually, the ramification divisor. It is of degree 
$3 m-3$, and its direct image $f_{*} R$ is, by the projection formula, of degree $3 m^{2}-3 m$. Write $f_{*} R=a D+D^{\prime}$ where $a \geq 0$ and $D^{\prime}$ does not contain $D$ as a component. We shall show that the number $a$ is rather big.

Indeed, let us compute the intersection number $D^{\prime} \cdot D$ : on the one hand, it is equal to

$$
\left(f_{*} R-a D\right) \cdot D=9 m^{2}-9 m-9 a,
$$

on the other hand, by Proposition 1 , the multiplicity of $D^{\prime}$ at $P_{1}$ is at least $m^{2}-1-2 a$ and the multiplicity of $D^{\prime}$ at $P_{i}, 2 \leq i \leq 9$, is at least $m^{2}-1-a$. The intersection number is then at least $8\left(m^{2}-1-a\right)+2\left(m^{2}-1-2 a\right)$, so that $9 m^{2}-9 m-9 a \geq$ $10 m^{2}-10-12 a$, or,

$$
a \geq \frac{m^{2}+9 m-10}{3}
$$

Now if we have ten points in which $f$ is completely ramified, they are not all on a cubic, so there are ten cubics $D_{1}, \ldots, D_{10}$, each $D_{i}$ containing exactly nine points of our ten, and each being a component of $f_{*} R$ of multiplicity at least $\frac{m^{2}+9 m-10}{3}$. But since $10\left(m^{2}+9 m-10\right)>3 m^{2}-3 m$, this is impossible.

Remark: We see that we have not completely generalized the argument from the Introduction: that is, we have not found a "Hurwitz formula" (that is, a statement similar to Lemma 2 and Lemma 3) for differentials on a non-reduced curve and so in our proof we have to circumvent the case where $C=f^{*} D$ is non-reduced. If $D$ is smooth, we do it by considering $C_{\text {red }}$, but if it is not, then there are examples where the contribution of a point of complete ramification to $\operatorname{deg}\left(\omega_{C_{\text {red }}}\right)$ is not sufficient for our purposes (for instance, zero, as in our examples from the previous section). Anyway, even if we could understand those differentials better, this would only give an upper bound of 8 points. It is of course impossible to prove by our method that there are at most three. On the other hand, this could considerably improve our Theorem 2 , giving immediately a bound $2 N^{2}$, whereas in what follows we obtain rapidly $6 N^{2}$ and can lower it to $4 N^{2}$ only with some effort.

\section{Generalizing to higher dimension}

Let $f: \mathbb{P}^{N-1} \rightarrow \mathbb{P}^{N-1}, N>3$, be a morphism of degree bigger than one, and $f^{*} \mathcal{O}(1)=\mathcal{O}(m)$. Let $L_{1}, \ldots L_{k}$ be codimension-two subspaces of $\mathbb{P}^{N-1}$ such that for every $1 \leq i \leq k, f^{-1}\left(L_{i}\right)=L_{i}$. Notice that, contrary to the situation of the last section, we suppose that our linear subspaces are completely invariant (rather than have another linear subspace as the inverse image). This is because we will need to assume that $m$ is sufficiently big with respect to $N$ ( compare with the proof of Proposition 2 ). If we want to estimate the number of completely invariant linear subspaces, we may of course assume this, iterating $f$ if necessary.

Consider a general $S=\mathbb{P}^{2} \subset \mathbb{P}^{N-1}$. By a Bertini-type theorem, the inverse image $T$ of this $\mathbb{P}^{2}$ is a smooth surface; obviously it is a complete intersection of 
type $(m, m, \ldots, m)$, of degree $m^{N-3}$. Let $P_{i}$ be the intersection point of $S$ and $L_{i}$. The degree of the map from $f^{-1}\left(L_{i}\right)$ to $L_{i}$ is $m^{N-3}$, and the local degree of $f$ at a general point of $f^{-1}\left(L_{i}\right)$ is $m^{2}$. We see thus that each $P_{i}$ has $m^{N-3}$ points $Q_{i, j} \in T, j=1, \ldots, m^{N-3}$, in its inverse image, and the local degree of $f_{T}$ (the restriction of $f$ to $T$ ) at those points is $m^{2}$. In what follows we shall get a bound for $k$ by studying the map $f_{T}: T \rightarrow S=\mathbb{P}^{2}$.

Let us introduce some more notations: denote by $E$ the set of $P_{i}, 1 \leq i \leq k$, and by $H_{T}$ the hyperplane section class on $T$. The canonical class $K_{T}$ of $T$ is thus $(-N+(N-3) m) H_{T}$, and the ramification divisor of $f_{T}$ is $(m-1) N H_{T}$ (so that $f_{*} R$ is of degree $\left.m^{N-2}(m-1) N\right)$.

Here is an analogue of Proposition 2, adapted to our purposes:

Proposition 3: In the situation as above, let $D$ be a (reduced) curve of degree d on $S=\mathbb{P}^{2}$. If $m>>N$, then

a) $D$ has at most $N d-1$ points of $E$ in its smooth locus;

b) If $D$ does not contain any component of the branch locus, then there are at most $N d-1$ points of $E$ on $D$;

c) There are at most $N d+\frac{(d-1)(d-2)}{2}-1$ points of $E$ on $D$.

Proof: a) Suppose that there are $N d$ or more points of $E$ in the smooth locus of $D$. Let $C$ be the (scheme-theoretic) inverse image of $D$ under $f_{T}$. $C$ is a curve cut out on $T$ by an hypersurface of degree $m d$, and of course it need not be reduced; let $C_{r e d}$ denote its reduction. Its dualizing sheaf $\omega_{C_{\text {red }}}$ is a line bundle because $C_{\text {red }}$ is a divisor on a smooth surface $T$. We cannot directly compute the degree of $\omega_{C_{\text {red }}}$, because, contrary to the case $N=3$, we cannot be sure that $C_{\text {red }}$ is a complete intersection. But we can get an inequality from the Hodge index theorem. Indeed, let $c=\frac{C_{r e d} H_{T}}{m^{N-3}}$ (if $C_{r e d}$ was a complete intersection, it would of course mean that $C_{\text {red }}$ is cut out on $T$ by an hypersurface of degree $c$ ). By the projection formula, we have that the degree of the map from $C_{r e d}$ to $D$ is $\frac{m c}{d} m^{N-3}$. Now by the Hodge index theorem, $C_{r e d}^{2} H_{T}^{2} \leq\left(C_{r e d} H_{T}\right)^{2}$, that is, $C_{r e d}^{2} \leq c^{2} m^{N-3}$. Finally, we have:

$$
\operatorname{deg}\left(\omega_{C_{r e d}}\right)=K_{T} C_{r e d}+C_{r e d}^{2} \leq(-N+c+(N-3) m) c m^{N-3},
$$

and of course

$$
\operatorname{deg}\left(\omega_{D}\right)=(d-3) d
$$

Denote now by $e_{i, j}$ the local degree of the map from $C_{r e d}$ to $D$ at those $Q_{i, j}$ which are on $C_{\text {red }}$ (if $C$ was reduced, we would of course have $e_{i, j}=m^{2}$ ). Obviously we have:

$$
\sum_{j=1}^{m^{N-3}} e_{i, j}=\frac{m c}{d} m^{N-3}
$$

for all $i$ such that $P_{i}$ is on $D$. By Lemma 2, if $P_{i}$ is a smooth point of $D$, then at each of the corresponding points $Q_{i, j}$, the length of the cokernel of the injection of the dualizing sheaves is at least $e_{i, j}-1$. 
This, and the assumption that there are at least $N d$ points of $E$ in the smooth locus of $D$, implies the following inequality:

$$
(*)(-N+c+(N-3) m) c m^{N-3} \geq \frac{m c}{d} m^{N-3} d(d-3)+N d m^{N-3}\left(\frac{m c}{d}-1\right),
$$

which reduces to

$$
c^{2}-N c \geq m c d-N d .
$$

This inequality, as in the proof of Theorem 1, cannot hold if $m c-N>0$.

As was already said, we assume that the subspaces $L_{i}$ are completely invariant, that is, $f^{-1}\left(L_{i}\right)$ is not an arbitrary linear subspace, but $L_{i}$ itself, and so we may assume that $m$ is arbitrarily big with respect to $N$, replacing $f$ by a suitable power of $f$. The number $c$ however is not necessarily an integer and depends on the map $f$ and the curve $D$, so we need to make an estimation for $c$ to conclude. In fact it is not difficult to bound $c$ from below as follows: the ramification divisor of our original map $f: \mathbb{P}^{N-1} \rightarrow \mathbb{P}^{N-1}$ is a (possibly reducible and non-reduced) hypersurface of degree $m N-N$. Therefore, at a general point of any component of this divisor, the local degree of $f$ is at most $m N-N+1$, and the image of the set of points where this local degree is bigger, is a subvariety of codimension at least two in $\mathbb{P}^{N-1}$. Since our surface $S$ is a general plane in $\mathbb{P}^{N-1}$, we can choose it so that it intersects this subvariety in a finite set of points. For such a choice of $S$, and any reduced curve $D \subset S$, the multiplicity of any irreducible component of $C=f^{*} D$ is at most $m N-N+1$. This gives

$$
c \geq \frac{m d}{m N-N+1}
$$

and, taking for example $m \geq N^{2}$, we obtain $m c>N$. This proves the part a).

b) The condition that $D$ does not contain a component of the branch locus means that $f^{*} D$ is reduced. So one could repeat a part of the above argument applying Lemma 3 instead of Lemma 2 and remarking that now $c=m d$, so things become easier. But it is probably better to apply Proposition 1 directly. Indeed, $f_{*} R$ is, by projection formula, of degree $m^{N-2}(m-1) N$. Suppose that $D$ passes through $r$ points of complete ramification. By Proposition 1, these are points of multiplicity at least $\left(m^{2}-1\right) m^{N-3}$ on $f_{*} R$. One has then $m^{N-2}(m-1) N d=f_{*} R \cdot D \geq$ $r\left(m^{2}-1\right) m^{N-3}$, so $r<N d$.

c) If $D$ is irreducible, this is just a reformulation of a), since $\frac{(d-1)(d-2)}{2}$ is the arithmetic genus $p_{a}(D)$ of $D$, so $D$ cannot have more than $\frac{(d-1)(d-2)}{2}$ singularities. Otherwise, $D=\bigcup D_{i}$ with $D_{i}$ irreducible of degree $d_{i}$. Each $D_{i}$ contains less than $N d_{i}+p_{a}\left(D_{i}\right)$ points of complete ramification. Their union $D$ contains then less than $N d+p_{a}(D)$ of those points, as follows from the inequality $p_{a}(D) \geq \sum p_{a}\left(D_{i}\right)$ (implied by standard exact sequences).

Notice that, as in the previous section, Proposition 3 implies almost immediately that $|E|<6 N^{2}$ : 
Proposition 4: Let $N$ be a natural number and $E$ a set of points in the plane, with the following properties:

(1) Any curve of degree $d$ contains at most $N d-1$ points of $E$ in its smooth locus;

(2) Any "flexible" curve (that is, a general member of a linear system without base components) of degree $d$ contains at most $N d-1$ points of $E$.

Then $E$ has less than $6 N^{2}$ points.

Proof: Suppose this is not true. Fix a subset $V \subset E$ of $6 N^{2}$ points and consider the linear system of curves of degree $4 N$ containing $V$. The dimension of this linear system is at least $4 N(4 N+3) / 2-6 N^{2}=2 N^{2}+6 N$. Denote by $l$ the degree of the union of its base components, i.e. of its fixed part. Remark that $l$ cannot be very big: indeed, the degree of the free part is $4 N-l$ and so the dimension of our linear system is at most $(4 N-l)(4 N-l+3) / 2$, so we get an inequality $(4 N-l)(4 N-l+3) \geq 4 N^{2}+12 N$, or $12 N^{2}-8 N l+l^{2} \geq 0$, from where $l \leq 2 N$. Now the property (1) implies that our fixed part contains at most $N l+l(l-3) / 2$ points of $E$ (the same argument as in Proposition $3 \mathrm{c}$ )). Since $l \leq 2 N$, this is at most $2 \mathrm{Nl}$. A general member of the free part, by the property (2), contains less than $N(4 N-l)$ points of $E$. So a general curve of our linear system contains less than $2 N l+N(4 N-l)=4 N^{2}+N l \leq 6 N^{2}$ points of $E$, a contradiction.

Remarks: 1) For small values of $N$, one obtains a better bound by the same type of arguments. An interested reader can verify that for $N=4$, by taking sextics through 24 points of $E$ and applying Proposition 3, we obtain $|E|<24$ (and of course for $N=3$, by taking quartics through 12 points of $E$, we obtain $|E|<12$ ).

2) (one of possible proofs of Theorem 2:) With a more elaborate argument of this kind, involving a Cayley-Bacharach type statement, we arrive to the bound of Theorem 2, that is, $4 N^{2}$. This can be done as follows: suppose that $E$ has $4 N^{2}$ or more points, fix a subset $V$ of $4 N^{2}$ points and consider curves of degree $4 N$ containing $V$. This is a linear system of dimension at least $4 N^{2}+6 N$. In the same way as above, we see that the degree $l$ of its fixed part is less than $\frac{4}{3} N$. The fixed part contains less than $N l+\frac{(l-1)(l-2)}{2}$ points of $V$, and so on the free part there is a set $V^{\prime}$ of at least $4 N^{2}-N l-\frac{l(l-3)}{2}$ of them. This is not enough to get a contradiction immediately, but let us remark that the conditions imposed by the points of $V^{\prime}$ on curves of degree $4 N-l$ are far from being independent. Indeed, the number of those conditions is at most $\frac{(4 N-l)(4 N-l+3)}{2}-4 N^{2}-6 N=4 N^{2}-4 N l+\frac{l(l-3)}{2}$, whereas the number of points in $V^{\prime}$ is at least $4 N^{2}-N l-\frac{l(l-3)}{2}$. When $l<\frac{4}{3} N$, the number of conditions is clearly inferior to the number of points: we obtain that no collection of $4 N^{2}-4 N l+\frac{(l-1)(l-2)}{2}$ points of $V^{\prime}$ imposes independent conditions on curves of degree $4 N-l$. At this point, let us recall the following result from [EP] (Corollaire $2)$ : 
Let $M$ be a set of $A$ points in the plane, which do not impose independent conditions on curves of degree $\tau$. Let $s$ be a positive integer such that $s^{2} \leq A$ and $\tau>s-3+\frac{A}{s}$. Then there exists an integer $t, 0<t<s$, and a subset $M^{\prime} \subset M$ consisting of at least $t(\tau-t+3)$ points, which is contained in a curve of degree $t$.

Straightforward calculations show that this result applies to $A=4 N^{2}-4 N l+$ $\frac{(l-1)(l-2)}{2}, \tau=4 N-l$ and $s=[\sqrt{A}]$, and, moreover, that for any $0<t<s$, we have $t(\tau-t+3) \geq N t+\frac{(t-1)(t-2)}{2}$. Therefore there is a curve of degree $t$ containing at least $N t+\frac{(t-1)(t-2)}{2}$ points of $V$. But this contradicts Proposition 3, so we are done: $E$ contains less than $4 N^{2}$ elements.

It is also possible to give an elementary and self-contained proof of Theorem 2 . This is what we shall do in the next section.

\section{Points of high multiplicity on plane curves}

Recall that, for example, in the proof of Theorem 1, it was an essential observation (resulting from Proposition 1) that a point of complete ramification is a point of multiplicity at least $m^{2}-1$ on the divisor $f_{*} R$ of degree $3 m^{2}-3 m$. Remark that if our $R$ was irreducible, or even reduced, this would imply without any further work that $f$ is completely ramified at 8 points at most. Indeed, a simple calculation shows that a reduced curve of degree $3 m^{2}-3 m$ cannot have more than 8 points of multiplicity $m^{2}-1$ or more (provided that $m>1$, of course). In general, let $s>1$ be an integer, and denote by $E_{s}(B)$ the set of points of multiplicity at least $s$ on a reduced plane curve $B$ of degree $b$. The number of elements in $E_{s}(B)$ can be bounded by a function depending only on the ratio $\frac{b}{s}$. Indeed, a point of multiplicity at least $s$ lowers the geometric genus of a curve by at least $s(s-1) / 2$, and the lowest possible geometric genus of a reduced curve of degree $b$ is $1-b$, so that we get an inequality $\left|E_{s}(B)\right| s(s-1) / 2 \leq(b-1)(b-2) / 2+1-b$, or $\left|E_{s}(B)\right| \leq \frac{b(b-1)}{s(s-1)}$, and we can roughly bound this, say, by $2\left(\frac{b}{s}\right)^{2}$.

Of course $f_{*} R$ is not necessarily reduced, and for an arbitrary effective divisor $B=\sum a_{i} B_{i}$ of degree $b$ in the plane, the observation above fails completely. One trivial reason for this is that $B$ can have a component of multiplicity $s$, and then $E_{s}(B)$ is an infinite set. But even if we suppose that it does not, there are still some obvious counterexamples. For instance, $B$ might be the union of a line of multiplicity $s-1$ and $b-s+1$ other lines. Such a configuration can have $b-s+1$ points of multiplicity $s$, and this is not bounded by a function of $\frac{b}{s}$.

However one can ask if such a bound holds if in addition we suppose that the low-degree components of $B$ contain "not too many" points of $E_{s}(B)$. The following proposition provides some bound under a very strong restriction of this kind. Together with Proposition 3, it implies Theorem 2.

Proposition 5: Let $B=\sum a_{i} B_{i}$ be an effective divisor of degree $b$ in the plane, and fix a subset $E_{s}$ of the set of points of multiplicity at least $s$ on $B$. Set $N=$ 
$\left[\frac{b}{s}\right]+1$. Suppose that each irreducible component $B_{i}$ of degree $\leq \frac{b}{s}$ contains at most $N \operatorname{deg}\left(B_{i}\right)-1$ points of $E_{s}$ in its smooth locus. Then $\left|E_{s}\right|<4 N^{2}$.

Remarks: 1) The notation " $B$ " comes from the "branch divisor". If $B$ is $f_{*} R$ from the last section and $s$ is the lower bound for the multiplicity at $P_{i}$ coming from Proposition 1 , then $\left[\frac{b}{s}\right]$ is the dimension of the ambient projective space, that is, our $N$ coincides with that of the last section.

2) We have to consider an arbitrary subset of the set of points of multiplicity at least $s$ on $B$, rather than this whole set, because we want to deduce Theorem 2 from this proposition by taking the set of $P_{i}^{\prime}$ 's for $E_{s}$. Of course on $B=f_{*} R$ there can be other points of high multiplicity than the points $P_{i}$. The assumption of the proposition implies immediately that $E_{s}$ is finite: indeed, on any component which might be of multiplicity $s$ or more, there is only a finite number of elements of $E_{s}$.

The proof of this proposition is based on Lemma 5 below. Let us first introduce some more notations. Consider an irreducible plane curve $D$ of degree $d$, and let $h$ be a function on $D \cap E_{s}$, taking values in the interval $[0,1]$. For an integer $r \geq 0$, define

$$
q_{r, h}(D)=\sum_{y \in D \cap E_{s}} h(y)\left(\text { mult }_{y}(D)\right)^{r} .
$$

Write $B=a D+D^{\prime}$, where $a \geq 0$ and $D^{\prime}$ is an effective divisor not containing $D$.

Lemma 5: For any $h$ as above, we have an inequality

$$
b d-s q_{1, h}(D) \geq a\left(d^{2}-q_{2, h}(D)\right) .
$$

Proof: The intersection number $D^{\prime} D$ is equal to $(b-a d) d$. On the other hand,

$$
\begin{gathered}
D^{\prime} D \geq \sum_{y \in D \cap E_{s}} \text { mult }_{y}\left(D^{\prime}\right) \text { mult }_{y}(D) \geq \sum_{y \in D \cap E_{s}} h(y)\left(\text { mult }_{y}(B)-\text { mult }_{y}(D) a\right) \text { mult }_{y}(D) \geq \\
\geq \sum_{y \in D \cap E_{s}} h(y)\left(s-\text { mult }_{y}(D) a\right) \text { mult }_{y}(D)=s q_{1, h}(D)-a q_{2, h}(D),
\end{gathered}
$$

and our inequality follows.

Now let $q_{\text {reg }}(D)$ (resp. $q_{\text {sing }}(D)$ ) denote the number of smooth (resp. singular) points of $D$ which are in $E_{s}$. Let $\mu(D)$ denote the sum $\sum_{x \in \operatorname{Sing}(D) \cap E_{s}} \operatorname{mult}_{x}(D)$. One checks easily that $\mu(D)<d^{2}$ and that $q_{\text {sing }}(D) \leq \mu(D) / 2<d^{2} / 2$.

Lemma 5 has the following long 
Corollary: a) If $D$ is not a component of $B$, then $\left|D \cap E_{s}\right| \leq \frac{b d}{s}$;

b) If $D$ is a component of $B$ and $d>\frac{b}{s}$, then $q_{\text {reg }}(D) \leq \frac{b d}{s}$;

c) Let $I$ be any subset of $D \cap E_{s}$, such that $\sum_{x \in I}$ mult $_{x}(D) \leq d^{2}$. Then $|I| \leq \frac{b d}{s}$. In particular, $q_{\text {sing }}(D) \leq \frac{b d}{s}$.

d) If $q_{\text {reg }}(D)<d^{2}$, then $\frac{q_{r e g}(D)}{2}+q_{\text {sing }}(D) \leq \frac{b d}{s}$.

Proof: a) In this case $a=0$. The assertion follows from Lemma 5 if we take the function $h \equiv 1$ and remark that $q_{1, h}(D) \geq\left|D \cap E_{s}\right|$.

b) Let $h$ be equal to one on smooth points of $D$ and to zero on singular points of $D$. Then $q_{1, h}(D)=q_{2, h}(D)=q_{\text {reg }}(D)$, and the inequality of Lemma 5 reads as follows: $(b-a d) d \geq(s-a) q_{\text {reg }}(D)$. Remark that by assumption $d>\frac{b}{s}$, so $D$ is of multiplicity less than $s$ in $B$, so $s-a>0$. If we suppose that $q_{\text {reg }}(D)>\frac{b d}{s}$, we obtain that $(b-a d)>(s-a) \frac{b}{s}$, or, since $a \neq 0$, that $d<\frac{b}{s}$, contradicting the assumption.

c) Take $h=\frac{1}{\text { mult }_{y}(D)}$ if $y \in I$, and zero otherwise. Lemma 5 gives then $b d-s|I| \geq$ 0, q.e.d.

d) If $q_{\text {reg }}(D)+\mu(D) \leq d^{2}$, then by c), $\left|D \cap E_{s}\right| \leq \frac{b d}{s}$ and the assertion is obvious, so we may assume that $q_{r e g}(D)+\mu(D)>d^{2}$. Consider the number $t=\frac{d^{2}-q_{r e g}(D)}{\mu(D)}$, by assumption we have then $0<t<1$. So Lemma 5 applies to the following function $h: h(y)=\frac{t}{\text { mult }_{y}(D)}$ if $y$ is singular on $D$ and $h(y)=1$ if $y$ is smooth on $D$. The inequality of Lemma 5 rewrites as

$b d-s\left(q_{\text {reg }}(D)+t q_{\text {sing }}(D)\right) \geq a\left(d^{2}-q_{\text {reg }}(D)-t \mu(D)\right)=0$,

which gives

$$
\frac{b d}{s} \geq q_{r e g}(D)+\frac{d^{2}-q_{r e g}(D)}{\mu(D)} q_{\text {sing }}(D)=q_{\text {reg }}(D)\left(1-\frac{q_{\text {sing }}(D)}{\mu(D)}\right)+q_{\text {sing }}(D) \frac{d^{2}}{\mu(D)} .
$$

Our assertion follows now from the remarks made just before the Corollary: $\mu(D)<$ $d^{2}$ and $q_{\text {sing }}(D) \leq \mu(D) / 2$.

Proof of Proposition 5: First let us show that the assumption, together with the Corollary, implies that any curve $D$ of degree $d$ contains less than $\frac{3}{2} N d$ points of $E_{s}$. Indeed, we may suppose that $D$ is irreducible; if $d>\frac{b}{s}$, then by a) and b) of the Corollary, we have $q_{\text {reg }}(D) \leq \frac{b d}{s}<d^{2}$, that is, we can apply d). And this implies that $\left|D \cap E_{s}\right|=\frac{1}{2} q_{\text {reg }}(D)+\left(\frac{1}{2} q_{\text {reg }}(D)+q_{\text {sing }}(D)\right) \leq \frac{3}{2} \frac{b d}{s}<\frac{3}{2} N d$ as required.

If $d \leq \frac{b}{s}$, then $q_{\text {reg }}(D)<N d$, by assumption if $D$ is a component of $B$ and by a), if not. It remains to remark that $q_{\text {sing }}(D)<d^{2} / 2 \leq \frac{b d}{2 s}$, so again $\left|D \cap E_{s}\right|<\frac{3}{2} N d$.

Also, recall that the Corollary says that a curve of degree $d$ which does not have common components with $B$, contains at most $\frac{b d}{s}$ (so less than $N d$ ) points of $E_{s}$.

From these two facts we deduce $\left|E_{s}\right|<4 N^{2}$ : indeed, suppose that $E_{s}$ has $4 N^{2}$ or more elements. Consider the curves of degree $3 N$ passing through $4 N^{2}$ points of $E_{s}$. It is a linear system of dimension at least $\frac{3 N(3 N+3)}{2}-4 N^{2}$. Let $l$ be the 
degree of the union of its base components. The degree of the free part is then $3 N-l$, and the dimension of the linear system is at most $\frac{(3 N-l)(3 N-l+3)}{2}$, so we get an inequality $N^{2}+9 N \leq(3 N-l)(3 N-l+3)$, from which one deduces $l \leq 2 N$. Now the free part contains at most $\frac{b}{s}(3 N-l)$ points of $E_{s}$, because its general member does not contain any component of $B$. The fixed part contains less than $\frac{3}{2} N l$ points of $E_{s}$. So we conclude that a general curve of our linear system contains less than $N(3 N-l)+\frac{3}{2} N l=3 N^{2}+\frac{1}{2} N l \leq 4 N^{2}$ points of $E_{s}$, and this is a contradiction. Proposition 5 is proved.

Proof of Theorem 2: Let us recall the situation: we have a finite morphism $f_{T}$ : $T \rightarrow \mathbb{P}^{2}$, where $T$ is a smooth complete intersection surface of type $(m, m, \ldots, m)$ in $\mathbb{P}^{N-1}$, and $f^{*} H_{\mathbb{P}^{2}}=m H_{T}$ where $H$ stands for the hyperplane section divisor. We suppose that $m>>N$, so that Proposition 3 applies. By adjunction and Hurwitz' formula, we compute the ramification divisor $R$ of $f_{T}: R$ is linearly equivalent to $(m-1) N H_{T}$. By projection formula, $f_{*} R$ is a divisor of degree $m^{N-2}(m-1) N$. Furthermore, we have a set $E$ of points $P_{i}, 1 \leq i \leq k$, on $\mathbb{P}^{2}$, and we know that the inverse image of each $P_{i}$ consists of $m^{N-3}$ points on $T$, and in each of those, the local degree of the map $f_{T}$ is $m^{2}$.

By Proposition $1, f_{*} R$ is of multiplicity at least $m^{N-3}\left(m^{2}-1\right)$ at $P_{i}$, for any $1 \leq i \leq k$. It remains to apply Proposition 5 to $B=f_{*} R, b=m^{N-2}(m-1) N$, $s=m^{N-3}\left(m^{2}-1\right)$ and $E_{s}=E$. Indeed, Proposition 3 implies that the condition of Proposition 5 is satisfied.

\section{References}

[BPV] W. Barth, C. Peters, A. Van de Ven: Compact complex surfaces, SpringerVerlag, 1984.

[BD] J.-Y. Briend, J. Duval: Deux caractérisations de la mésure d'équilibre d'un endomorphisme de $\mathbb{P}^{k}(\mathbb{C})$, Publ. Math. IHES 93 (2001), 145-159.

[BCS] J.-Y. Briend, S. Cantat, M. Shishikura: Linearity of the exceptional set for maps of $\mathbb{P}^{k}(\mathbb{C})$, preprint.

[CLN] D. Cerveau, A. Lins Neto: Hypersurfaces exceptionnelles des endomorphismes de $\mathbb{C P}^{n}$, Bol. Soc. Brasil. Mat. (NS), 31 (2000), 155-161.

[EP] Ph. Ellia, C. Peskine: Groupes de points de $\mathbb{P}^{2}$ : caractère et position uniforme, in: Algebraic geometry, l'Aquila 1988, Lecture Notes in Maths. 1417.

[FS] J. Fornaess, N. Sibony: Complex dynamics in higher dimension I, Asterisque 222 (1994), 201-231. 